\title{
Optical waveguide mode control by nanoslit-enhanced terahertz field
}

Novitsky, Andrey; Zalkovskij, Maksim; Malureanu, Radu; Jepsen, Peter Uhd; Lavrinenko, Andrei

Published in:

Optics Letters

Publication date:

2012

Document Version

Publisher's PDF, also known as Version of record

Link back to DTU Orbit

Citation $(A P A)$ :

Novitsky, A., Zalkovskij, M., Malureanu, R., Jepsen, P. U., \& Lavrinenko, A. (2012). Optical waveguide mode control by nanoslit-enhanced terahertz field. Optics Letters, 37(18), 3903-3905.

\section{General rights}

Copyright and moral rights for the publications made accessible in the public portal are retained by the authors and/or other copyright owners and it is a condition of accessing publications that users recognise and abide by the legal requirements associated with these rights.

- Users may download and print one copy of any publication from the public portal for the purpose of private study or research.

- You may not further distribute the material or use it for any profit-making activity or commercial gain

- You may freely distribute the URL identifying the publication in the public portal

If you believe that this document breaches copyright please contact us providing details, and we will remove access to the work immediately and investigate your claim. 


\title{
Optical waveguide mode control by nanoslit-enhanced terahertz field
}

\author{
A. Novitsky, ${ }^{1,2}$ M. Zalkovskij, ${ }^{1}$ R. Malureanu, ${ }^{1}$ P. U. Jepsen, ${ }^{1}$ and A. V. Lavrinenko ${ }^{1, *}$ \\ ${ }^{1}$ DTU Fotonik-Department of Photonics Engineering, Technical University of Denmark, \\ Ørsteds Pl. 343, DK-2800 Kgs. Lyngby, Denmark \\ ${ }^{2}$ e-mail: andrey.novitsky@tut.by \\ *Corresponding author: alav@fotonik.dtu.dk
}

Received July 4, 2012; accepted July 31, 2012;

posted August 3, 2012 (Doc. ID 171706); published September 14, 2012

\begin{abstract}
In this Letter we propose a scheme providing control over an optical waveguide mode by a terahertz (THz) wave. The scheme is based on an optimization of the overlap between the optical waveguide mode and the THz field, with the THz field strength enhanced by the presence of a metallic nanoslit surrounding the waveguide. We find an optimum balance between the optical mode attenuation and Kerr-induced change in the propagation constant. The criterion for a $\pi / 2$-cumulative phase shift, for instance for application in a Mach-Zehnder interferometer configuration, requires $10 \mathrm{kV} / \mathrm{cm}$ THz field, which in turn is estimated to result in a nonlinear change of the refractive index in the waveguide of 0.001 . Our simulations prove that it is quite reasonable to observe the effect experimentally. (c) 2012 Optical Society of America

OCIS codes: $190.3270,190.4360$.
\end{abstract}

The idea of controlling light with another optical wave has received much attention in the past decades. Nonlinear interactions in suitable optical materials are employed to transfer information from one electromagnetic wave to another, and form the basis of most nonlinear optics. Of special interest here is the capability of influencing near-infrared (NIR) waves by $\mathrm{THz}$ fields. Currently, second-order techniques such as free-space electro-optic sampling [ $[\underline{-}-\underline{3}]$ and THz-induced lensing [4] are used for all-optical coherent detection of $\mathrm{THz}$ fields, and recently the third-order technique of airbiased coherent detection was introduced [5], with particular relevance for detection of intense, ultrabroadband $\mathrm{THz}$ transients.

Common to all optics-based detection techniques is the limited THz pulse energy available, and therefore, techniques for local enhancement of the $\mathrm{THz}$ field, for instance by plasmonic effects, are highly desirable. However, the majority of plasmonic phenomena are resonant and require sophisticated conditions in the $\mathrm{THz}$ range. Seo et al. demonstrated strong broadband enhancement of the THz field strength in a nanoslit [6]. Here we present a concept for a practical implementation of the use of this broadband field enhancement phenomenon in a device for manipulation of optical waves by a $\mathrm{THz}$ field.

The THz and optical fields operate at radically different wavelength scales. From a computational point of view it is resource-demanding to solve the problem of their interaction numerically. We split the problem into two parts, dealing with the THz and NIR frequency domain separately. In the $\mathrm{THz}$ frequency domain we compute the field distribution and local field enhancement in a nanoslit and estimate the THz-field induced change of the refractive index due to the nonlinearity of the material. The calculated nonlinear refractive index profile is then applied in a full-wave simulation of the optical mode propagation. CST Microwave Studio is used to perform simulations both in optical and $\mathrm{THz}$ ranges.

Terahertz modulation of the signals can be realized in the silicon-polymer hybrid systems as in [7]. It is an all-optical way based on the ultrafast Kerr effect in four-wave-mixing. In this Letter we adopt the MachZehnder design similar to [7] as the way of possible measurements (and validation) of the effect [Fig. 1(a)]. The design of the structure is shown in Fig. 1(b). The system consists of a silica substrate with deposited metal (gold) on top. A nanosized slit of width $w$ is introduced in the $z$ direction along the metal surface. The nanoslit is partly filled with a nonlinear material, here exemplified by the chalcogenide glass arsenic trisulfide, $\mathrm{As}_{2} \mathrm{~S}_{3}$.

For the sake of effective guiding, the chalcogenide glass does not end right after the slit, but has a height $Y$. Gold is modeled as a Drude metal with permittivity $\varepsilon(\omega)=1-\omega_{p}^{2} /\left(\omega^{2}+\mathrm{i} \gamma \omega\right)$, where $\omega_{p}=1.37 \times 10^{16} \mathrm{rad} / \mathrm{s}$ and $\gamma=40.7 \times 10^{12} 1 / \mathrm{s}$ are the plasma and collision frequencies, respectively [6], and $\omega=2 \pi f$ is the angular frequency.

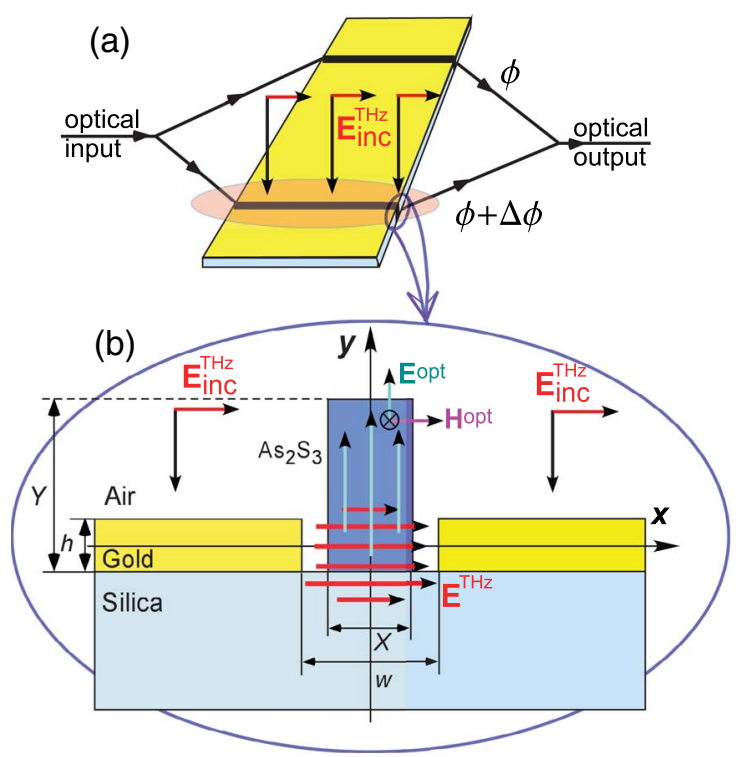

Fig. 1. (Color online) (a) Mach-Zehnder configuration for measurement of the nonlinear effect in (b) chalcogenide glass ridge waveguide embedded into a metallic nanoslit. 
The wavelengths of the THz and optical waves are $\lambda_{\mathrm{THz}}$ and $\lambda_{\text {opt }}$, respectively. The terahertz wave is incident on top of the metal film and is $x$-polarized. It creates a strong enhanced electric field [red arrows in Fig. 1(b)] in the nanoslit $[6,8]$. The NIR wave is localized in the high-index chalcogenide glass slab and propagates in the $z$ direction. Two modes are possible. The $x$-polarized field gives the strongest nonlinear coupling with the $\mathrm{THz}$ field due to the parallel polarizations and is confined inside the slit. However, this field also excites the surface plasmonpolaritons (SPPs) at the metallic edges $x= \pm w / 2$ and therefore rapidly decays due to strong attenuation. In contrast, the $y$-polarized optical mode [blue arrows in Fig. 1(b)] can have low propagation loss for small separations between the waveguide and the metal slit walls.

We model the Kerr-type nonlinear change of the refractive index of the chalcogenide glass as dependent on the mutual direction of $\mathrm{THz}$ and NIR polarizations. We introduce $n_{\|, \perp}^{\mathrm{opt}}=n_{0}^{\mathrm{opt}}+\Delta n_{\|, \perp}$ for the NIR polarization parallel and perpendicular to the terahertz one, where $\Delta n_{\|, \perp}=n_{2}^{\|}{ }^{\perp} \varepsilon_{0} c n^{\mathrm{opt}}\left|E_{x}^{\mathrm{THz}}\right|^{2} / 2, \varepsilon_{0}$ and $c$ are the vacuum dielectric permittivity and speed of light, respectively. We consider $n_{0}^{\mathrm{THz}}=n_{0}^{\mathrm{opt}}=2.7$ assuming the index in optics equals that in the low-THz regime [9].

According to THz-induced Kerr measurements by Hoffmann et al. [10] the Kerr coefficient in the THz range can be expected to be of similar value as in the NIR region. This gives us the possibility to estimate the magnitude of the incident THz field $\left|E_{\mathrm{inc}}^{\mathrm{THz}}\right|$ needed to get the required change of the refractive index, e.g., $\Delta n=$ 0.001 . Taking the nonlinear refractive index of $\mathrm{As}_{2} \mathrm{~S}_{3}$ with respect to the intensity as $n_{2}=1.1 \times 10^{-17} \mathrm{~m}^{2} / \mathrm{W}[\underline{11}]$ and assuming slit enhancement $G=100$ we get to

$$
\left|E_{\mathrm{inc}}^{\mathrm{THz}}\right|=\frac{1}{G} \sqrt{\frac{2 \Delta n}{n_{2} n_{0}^{\mathrm{opt}} \varepsilon_{0} c}}=17 \mathrm{kV} / \mathrm{cm} .
$$

Obtained field estimation is quite reasonable with stateof-the-art terahertz techniques [10], but powerful $\mathrm{THz}$ beams are still required.

The field distribution plays the decisive role in our scheme of controlling the NIR light propagation with a THz field. The THz field $E_{x}^{\mathrm{THz}}$ has a substantially flat field distribution along the $x$ axis, as shown in the inset of Fig. 2 for three different heights in the glass crosssections. Thus, we focus on the field distribution in the vertical direction. According to Fig. 2 the strongest $\mathrm{THz}$ field is pushed out of the nanoslit into the substrate due to the existing of air gaps with huge field there. The THz electric field in the chalcogenide waveguide decreases approximately exponentially from $y=-50 \mathrm{~nm}$ to $y=350 \mathrm{~nm}$ (red curve in Fig. 2). Therefore, the similar near-exponential distribution of the nonlinear refractive index $\Delta n$ is expected. The main $y$-component of the optical mode is concentrated inside $\mathrm{As}_{2} \mathrm{~S}_{3}$. It reaches maximum at about the middle of the $\mathrm{As}_{2} \mathrm{~S}_{3}$ slab (blue curve). To find the overlap region of the terahertz and optical fields we introduce the coupling coefficient $\kappa \sim \int_{-h / 2}^{Y-h / 2} \Delta n\left|E^{\mathrm{opt}}\right|^{2} \mathrm{~d} y$, in which the integrand $\Delta n\left|E_{\text {opt }}\right|^{2} \sim\left|E^{\mathrm{THz}}\right|^{2}\left|E^{\mathrm{opt}}\right|^{2}$ describes their interaction

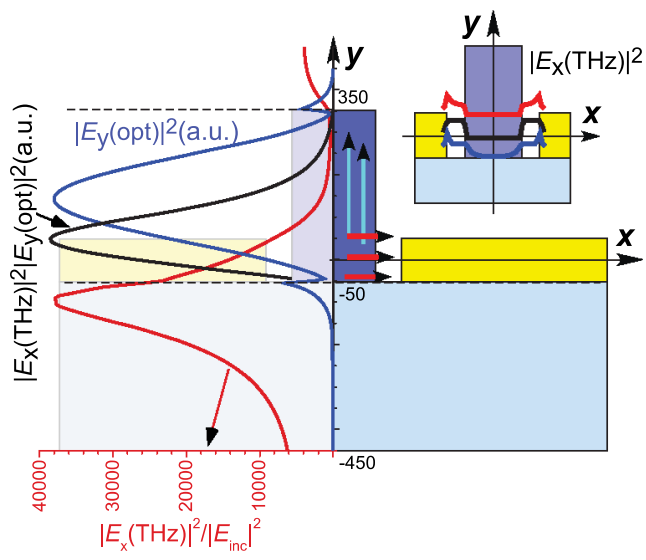

Fig. 2. (Color online) Field profile of the $\mathrm{THz}$ field $\left|E^{\mathrm{THz}}\right|^{2} /\left|E_{\mathrm{inc}}^{\mathrm{THz}}\right|^{2}\left(\lambda_{\mathrm{THz}}=1 \mathrm{~mm}\right.$, red curve), NIR mode $\left|E^{\mathrm{opt}}\right|^{2}$ ( $\lambda_{\text {opt }}=700 \mathrm{~nm}$, blue curve), and their interaction $\left|E^{\mathrm{THz}}\right|^{2}$ $\left|E^{\mathrm{opt}}\right|^{2}$ (black curve) at $x=0$. Parameters: $X=120 \mathrm{~nm}$, $Y=400 \mathrm{~nm}, w=200 \mathrm{~nm}$, and $h=100 \mathrm{~nm}$. In the inset, the terahertz field distributions in the $x$ direction for $y=-h / 2,0$, and $+h / 2$ are shown.

(black curve). The maximal interaction is achieved near the slit edge $y=h / 2$. The overlap could be increased by displacing the chalcogenide slab into the substrate, but the experimental realization of this design is extremely challenging. Since we want to demonstrate the feasibility of the effect in principle, we replace the realistic dependence of the induced change $\Delta n(y)$ with a step-like function of the height of constant $\Delta n$ equal $h+\Delta h$ [Fig. 3(a)].

We optimize the optical mode with respect to its losses, as shown in Fig. 3(b). The dielectric ridge waveguide has a clear optimal width $X$ of about $130 \mathrm{~nm}$. This value falls between two limiting cases. (i) When $X$ is small, the waveguide cannot confine the mode well, and (ii) when $X$ is large, the mode interacts strongly with metal. Loss optimization for the height $Y$ of the waveguide is evident, as shown in Fig. 3(c). The larger $Y$, the farther the mode is from the metal and the less is the attenuation. At the same time the NIR mode should be concentrated near the slit where the nonlinear processes happen. Therefore the geometrical parameters chosen for simulations are: $X=120 \mathrm{~nm}$ and $Y=400 \mathrm{~nm}$.

The performance of the device is determined by the overlap of the THz and optical modes and the attenuation of the optical mode. The overlap indicates how large the change is of the propagation constant due to the nonlinearity $\Delta \beta \sim \Delta n$, that is, how well the optical mode is localized in the nanoslit. However, the deeper the optical mode is localized in the metallic slit, the larger the losses. Therefore, we have a trade-off between the overlap and attenuation. To compare both parameters in a unified way we introduce dimensionless figure of merit (FOM) as the ratio of two distances, the length of the $\pi / 2$ phase shift $L_{\pi / 2}=\pi /(2 \Delta \beta)$ and the distance of the decrease of intensity of optical mode in $e$-times $D=1 /(2 \alpha)$ (remember $\left.I=I_{0} \exp (-2 \alpha D)\right)$. The FOM reads

$$
\mathrm{FOM}=\frac{L_{\pi / 2}}{D}=\frac{\pi \alpha}{\Delta \beta},
$$



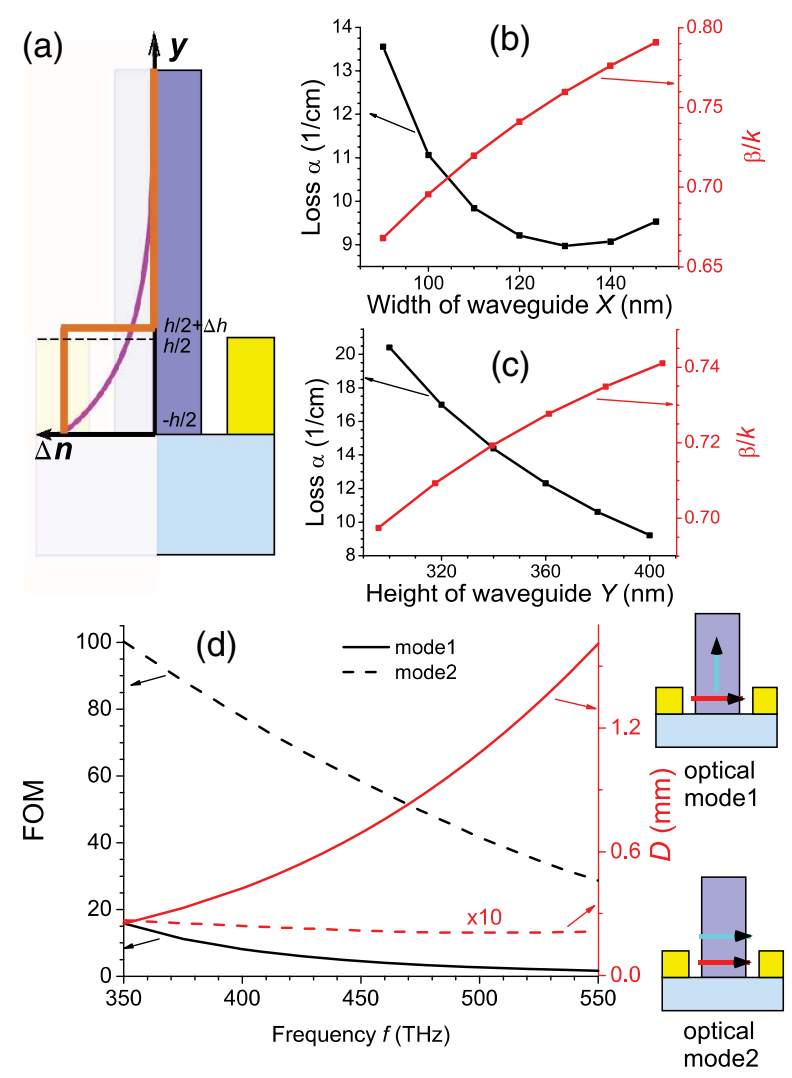

Fig. 3. (Color online) (a) Realistic nonlinear refractive index change (blue) is replaced by a step-like function (orange) in simulations. (b), (c) Field loss optimization of the geometrical parameters of waveguide (right scale shows the normalized propagation constant). (d) FOM (left) and distance $D$ of intensity decrease in $e$ times (right) for two optical modes (insets on the right-hand side). Parameters: $n_{2}^{\|}=0.001, \mathrm{n}_{\|}^{\perp}=n_{2}^{\|} / 4$, $h=100 \mathrm{~nm}$ and $w=200 \mathrm{~nm}, \Delta h=20 \mathrm{~nm}, \quad X=120 \mathrm{~nm}$, $Y=400 \mathrm{~nm}$.

where $\Delta \beta=\beta-\beta_{0}$ is the difference between propagation constants in the waveguide irradiated by THz fields $\beta$ and in the initial state $\beta_{0}, \alpha \approx \alpha_{0}$ is the field attenuation in the initial state. Apparently, the smaller FOM, the better, because smaller $L_{\pi / 2}$ and larger $D$ are preferred.

Assuming the anisotropic nonlinear response in the chalcogenide slab (largest along the terahertz field and four times smaller in the perpendicular direction) we calculated the FOM for the two optical modes (the number of four is chosen for example). In Fig. 3(d) it can be seen that the FOM of the $y$-polarized optical mode is much lower than that of the mode polarized along the THz field ( $x$-polarized) in spite of the four times smaller nonlinear coefficients for this polarization. The $y$-polarized optical mode is beneficial mainly due to low losses, so that the required effect can be reached owing to the longer propagation $\left(L_{\pi / 2}=3.2 \mathrm{~mm}, D=0.54 \mathrm{~mm}\right.$ at $\left.425 \mathrm{THz}\right)$. At high frequencies losses decrease more, because the mode is better concentrated in the dielectric $\mathrm{As}_{2} \mathrm{~S}_{3}$. Losses for the $x$-polarized mode are extremely large and almost do not depend on the frequency, because the main channel of attenuation is the excitation of SPPs at the slit edges.

In conclusion, we have demonstrated the possibility of controlling the propagation of an optical waveguide mode by a $\mathrm{THz}$ field. A ridge waveguide made from a nonlinear chalcogenide glass supports an optical mode manipulated by the nonlinearity caused by the THz radiation. We relax the requirement for high $\mathrm{THz}$ field intensities by positioning the waveguide in a nanosized slit in a thin metal film, resulting in a strong, local $\mathrm{THz}$ field enhancement. Matched oblique incidence of the $\mathrm{THz}$ wave can be used to ensure effective interaction of the $\mathrm{THz}$ and optical pulses over millimeter distances [12]. We anticipate the application of the results for the fast $\mathrm{THz}$ modulation of optical signals.

Danish Research Council for Technology and Production Sciences via project $\mathrm{THz} \mathrm{COW}$ is acknowledged.

\section{References}

1. Q. Wu and X. C. Zhang, Appl. Phys. Lett. 67, 3523 (1995).

2. P. U. Jepsen, C. Winnewisser, M. Schall, V. Schyja, S. R. Keiding, and H. Helm, Phys. Rev. E 53, R3052 (1996).

3. A. Nahata, D. H. Auston, T. F. Heinz, and C. J. Wu, Appl. Phys. Lett. 68, 150 (1996).

4. A. Schneider, I. Biaggio, and P. Gunter, Appl. Phys. Lett. 84, 2229 (2004).

5. J. Dai, X. Xie, and X. C. Zhang, Phys. Rev. Lett. 97, 103903 (2006).

6. M. A. Seo, H. R. Park, S. M. Koo, D. J. Park, J. H. Kang, O. K. Suwal, S. S. Choi, P. C. M. Planken, G. S. Park, N. K. Park, Q. H. Park, and D. S. Kim, Nature Photon. 3, 152 (2009).

7. M. Hochberg, T. Baehr-Jones, G. Wang, M. Shearn, K. Harvard, J. Luo, B. Chen, Z. Shi, R. Lawson, P. Sullivan, A. K. Y. Jen, L. Dalton, and A. Scherer, Nat. Mater. 5, 703 (2006).

8. A. Novitsky, M. Zalkovskij, R. Malureanu, and A. Lavrinenko, Opt. Commun. 284, 5495 (2011).

9. M. Zalkovskij, C. Z. Bisgaard, A. Novitsky, R. Malureanu, D. Savastru, A. Popescu, P. U. Jepsen, and A. V. Lavrinenko, Appl. Phys. Lett. 100, 031901 (2012).

10. M. C. Hoffmann, N. C. Brandt, H. Y. Hwang, K.-L. Yeh, and K. A. Nelson, Appl. Phys. Lett. 95, 231105 (2009).

11. B. J. Eggleton, B. Luther-Davies, and K. Richardson, Nat. Photonics 5, 141 (2011).

12. K. Iwaszczuk, A. Andryieuski, A. Lavrinenko, X. C. Zhang, and P. U. Jepsen, Appl. Phys. Lett. 99, 071113 (2011). 